\title{
Ethical Problems and Countermeasures of Artificial Intelligence Technology
}

\author{
$\mathrm{H} \mathrm{Y} \mathrm{Li}^{1}, \quad \mathrm{~J} \mathrm{~T} \mathrm{An}{ }^{2, *}$ and $\mathrm{Y}$ Zhang ${ }^{3}$ \\ ${ }^{1}$ School of Automation, Wuhan University of Technology \\ ${ }^{2}$ office of academic affairs, Wuhan University of Technology \\ ${ }^{3}$ School of Energy and Power Engineering, Wuhan University of Technology
}

\begin{abstract}
The in-depth development of artificial intelligence has brought great benefits and convenience to humankind. However, it also brings about ethical crises such as human rights, responsibility, generation, information, prejudice, which are worthy of our attention. Therefore, we should deepen the scientific and technological ethics under the guidance of Marxism, construct artificial intelligence ethical standards, strengthen the supervision of artificial intelligence, enhance the public ethical concepts, so that artificial intelligence can really cope with the risks and challenges under the guidance of scientific and technological ethics, and grasp the development opportunities of The Times.
\end{abstract}

\section{Introduction}

With the development of Internet, big data, cloud computing and other technology, the artificial intelligence algorithm with data of life elements and automatic decision-making of machine as the core has been more and more widely used. With the milestone event of AlphaGo's victory over the human top Go master Lee Sedol, artificial intelligence has created wide applications in finance, medical care, automatic driving, security, home and marketing, and brought great changes to the entire human society. At the same time, the boundary between the physical world and the individual is constantly blurred, which induces a series of ethical crises, and causes multiple obstacles to the development of social governance and structural system. [1]

The industry has long recognized the limitations of the famous "Three Laws of Robotics" devised by Asimov, a science-fiction writer. Some foreign research works, such as the EU Artificial Intelligence published by the European Commission and the Robot Ethics Charter issued by the Ministry of Industry, Commerce and Energy of Korea, focus on the ethical issues of artificial intelligence technology from certain perspectives, but no widely accepted ethical framework has been formed yet. In China, most scholars analyze and discuss the development history, superiority and limitation of artificial intelligence technology, as well as the relationship between artificial intelligence and human intelligence. However, there is still a lack of professional research on the ethical issues of artificial intelligence technology and corresponding countermeasures. [2]
The New Generation of Artificial Intelligence Development Plan released in 2017 put forward China's AI strategy, and the formulation of laws, regulations and ethical norms to promote the development of AI was proposed as an important guarantee measure. In July 2019, the Ninth Conference of the CPC Central Committee on Comprehensive Deepening Reform was held and a series of documents such as the Establishment Plan of the National Science and Technology Ethics Committee were released, indicating that China's science and technology undertaking is entering the process of ethically-guided development [3].

Therefore, it is necessary to deeply analyze the ethical issues caused by artificial intelligence technology, so that artificial intelligence can cope with risks and challenges under the guidance of science and technology ethics and develop scientifically and rationally.

\section{Main ethical problems caused by artificial intelligence technology}

\subsection{Ethical issues of human rights}

At present, using artificial intelligence technology, humans have been able to create artificial life that can perform simple tasks. With the development and progress of technology, more advanced artificial life will be born and evolve, and even produce human-like emotions and "humanity". At that point, we will have to decide whether they are right holders and whether -- and when -- they deserve the same rights, freedoms and protections as human beings. It can be clearly recognized that human beings treat or insult these objects inhumanly, which is a 
violation of human morality and ethics bottom line. Similarly, human rights violations by intelligent robots may also occur.

\subsection{Ethical issues of responsibility}

The ethics of responsibility is a holistic study of the responsibility of human society after considering the motivation, consequence and mode of the subject's behavior from the ethical perspective. It includes the identification of the relationship between the responsible parties, the judgment of responsibility, and the internal and external factors of responsibility and so on. [4]

Ethical problems arise due to the development of artificial intelligence technology, and its responsibility is the core issue in the research. The traditional responsibility system for technical consequences cannot cope with the uncertainty and negative results caused by the development and application of artificial intelligence technology. [5] For example, if the AI system has problems after being put into the hospital for consultation, the attribution of responsibility is a typical ethical problem of responsibility.

\subsection{Ethical issues of information}

The ethical requirements in the fields of information research and development, diffusion and application management are collectively referred to as information ethics.

In the era of big data with artificial intelligence, user data is in a state of transparency and de-privacy. The application of artificial intelligence technology has greatly expanded the scenarios, scope and quantity of personal information collection. The risk of invasion of privacy is greater, and there are more victims. In 2019, China Deep Net Vision Technology Co., Ltd. suffered a massive facial database information leak, exposing the highly sensitive personal privacy information of more than 2.56 million users. AI designers can sort out users' preferences and preferences based on their searches and then push relevant services to them, which interferes with people's freedom to deal with their private affairs. [6]

\subsection{Intergenerational ethical issues}

Intergenerational ethics is the general term of ethical relations and ethical norms between generations of human beings. It is an important part of social ethical relations and ethical forms, and has a profound impact on artificial intelligence technology. Intergenerational ethics mainly refers to the communication and generation gap under the guidance of moral values. Since artificial life is modelled after humans, society's intergenerational ethical system would collapse if artificial life were to evolve the same ability to reproduce as humans.

\subsection{Ethical issues of prejudice}

Sandra Warcher, a researcher in data ethics and algorithms at Oxford University, said: "The world is biased, historical data is biased, so it's not surprising that we get biased results." [7]

The Commission for Data Protection of the European Union and the Pew Research Center of the United States have released investigation and research reports on the discrimination of artificial intelligence algorithms, believing that the current artificial intelligence algorithms have produced discrimination of age, gender, race and other groups in the collection, classification, sorting, classification and interpretation of data. Amazon Group used AI recruitment software to review resumes between 2014 and 2017, but the software downgraded resumes that included the word "female" to contain a strong gender bias. [8]

\section{Main causes of ethical problems caused by artificial intelligence technology}

\subsection{Lack of public literacy and moral integrity}

Due to the lack of moral literacy and cultural cultivation, scientists or researchers, attracted by interests, violate professional ethics and conduct illegal and ethical research, which will pose a huge threat to the interests and safety of mankind. Most of these problems can be avoided if handled properly. Nevertheless, due to the public's lack of understanding of artificial intelligence technology, it is easy to misunderstand. When they hear about negative events or false information about artificial intelligence technology, they are easy to feel panic, resulting in rejection.

\subsection{Lack of ethical principles and regulations}

The main reason for the ethical problems caused by artificial intelligence is the lack of ethical principles and norms. Humans can vent their emotions to the robot, so that their emotions can be comforted and humans will become dependent on robots. However, robots cannot fully meet the needs of human communication. In the long run, it is easy to cause human depression, anxiety and other psychological problems.

\subsection{Policy and regulation lag and imperfection}

As an emerging technology, artificial intelligence lacks the restriction of relevant legal system. It can only be constrained by public opinion and technical means. However, neither of them is self-conscious and mandatory, so they cannot receive a good effect.

\subsection{Limitations of artificial intelligence technology}

There are still limitations to artificial intelligence, such as its ability to deal with human emotional mimicry. Artificial intelligence technology only executes the program set by human beings. It cannot have the feelings and thoughts of human beings, nor can it judge things or 
terminate actions by itself. If this limitation is used by illegal elements, it will cause serious consequences.

\subsection{Imperfect supervision mechanism}

Despite the rapid development of science and technology today, there is no effective supervision mechanism to supervise artificial intelligence technology, and no authority to regulate it. There are still many defects, such as the abuse of artificial intelligence technology.

\section{Countermeasures to the ethical problems of artificial intelligence technology}

\subsection{Deepen the ethics of science and technology under the guidance of Marxist thought}

Marx and Engels constructed technological thought and thinking science theory, which laid the ideological foundation for China's scientific and technological ethics. Marx's scientific and technological ethics first highly affirms the value of scientific and technological activities, and from the perspective of alienation, it is full of revolutionary democratic and dialectical colors. This is of guiding significance to the current subject of artificial intelligence. Marx's point of view based on human social development point of view, will be about science and technology as the basic elements of the society, from the productivity, social consciousness and social status is a fact of three kinds of human social Angle of view to discuss the relationship between technology and society, contains a profound historical dialectical materialist thoughts, to promote the development of artificial intelligence in our country ethics is of great significance. We should deepen the scientific and technological ethics of artificial intelligence development under the guidance of Marxism.

\subsection{Building ethical norms for artificial intelligence}

Constructing artificial intelligence ethical standards and standardizing ethical behaviors is an important basis for solving ethical problems. From the perspective of ethical norms, the relevant construction should be divided into two parts.

One is the design ethics of artificial intelligence. In current artificial intelligence research and development work, scientific exploration of the subject must be from the stage of product design, research and development and innovation, the responsibility system of ethics and technology of artificial intelligence evolution activity of artificial intelligence for judge inner dimension, enlarge the positive effects of artificial intelligence technology, as far as possible to reduce the negative impact of technology alienation to people and nature.

The other is the application ethics of artificial intelligence products. It is necessary to construct reasonable ethical system norms to regulate the owners and users of artificial intelligence. From the perspective of moral system norms, users will internalize them into the relevant beliefs of the subject itself in the actual use process. From the perspective of moral cultivation, the users of artificial intelligence need to strengthen their internal skills and their sense of responsibility in artificial intelligence products.

\subsection{Strengthen oversight of artificial intelligence}

Government supervision is an important guarantee to solve ethical problems. The potential threat of artificial intelligence should be fully considered. In traditional technology, the common way to prevent damage is to intervene after the harm has been done. However, for AI systems, A better approach would be to conduct immediate and ongoing ethical risk assessments. it is better to conduct immediate and ongoing moral hazard assessments, so that the artificial intelligence technology can be developed in a reasonable and effective way to prevent it from losing control.

In addition, AI-related industries should cooperate with the government and provide timely feedback to the government on the latest developments in the industry, including possible breakthroughs in technology in the near future.

\subsection{Enhance public ethics}

According to the schematic diagram of artificial intelligence ethical risk prevention framework proposed by the British ethicist Luciano Floridi [9], as shown in the Figure 1, the ethical risk responsibility of artificial intelligence includes the responsibility of designer, user and audience.

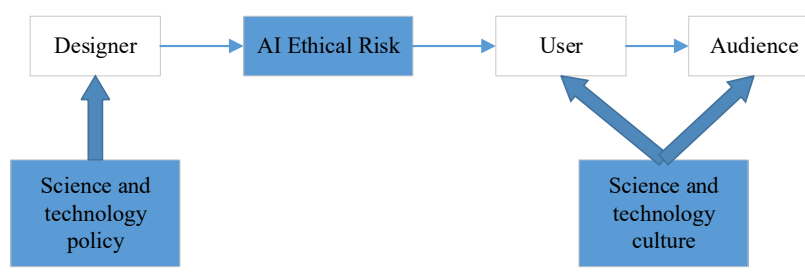

Fig 1. Schematic diagram of artificial intelligence ethical risk prevention framework

In the high-tech era, no one is a bystander. Only with the full coverage of ethical responsibility can the ethical risks of artificial intelligence be contained to the maximum extent. As a highly integrated product of technical attributes and social attributes artificial intelligence public cognition is an important factor for the implementation of AI ethical norms. More ways should be expanded to enhance the public's understanding of artificial intelligence technology and inspire and cultivate the public's ethical awareness. Firstly, public media should be used to create a good social atmosphere for the promotion of science and technology ethics, to help people distinguish right from wrong, true, good and beautiful, to resist wrong ideas and immoral behaviors, and to strengthen the attention of the whole society to ethical values. Secondly, starting from the characteristics 
of artificial intelligence neutral technology, let the public understand that the technology itself has no interests, encourage the public to keep rational to new things, rationally look at the issues related to artificial intelligence, so as to reduce the public's psychological anxiety and burden on this ethical issue.

\section{Conclusion}

With the deep development of modern artificial intelligence, its ethics has become a problem that we must pay attention to and examine. The ethical deviations such as human rights, responsibility, generation, information and prejudice caused by artificial intelligence gradually appear, which requires our ethical thoughts to develop continuously and adapt to them. The healthy and benign development of artificial intelligence and the guidance of science and technology ethics have a significantly positive correlation. Therefore, we should deepen the ethics of science and technology under the guidance of Marxism, the construction of artificial intelligence of ethics, to strengthen the regulation of artificial intelligence, enhance the public ethics, and make artificial intelligence can under the guidance of science and technology ethics really deal with risks and challenges. At the same time of preventing risks, attention should be paid to avoid the excessive restriction or the use of inappropriate risk reduction methods to curb the development of the industry.

\section{Acknowledgments}

This research was financially supported by the High Technology Ship Research project of the Ministry of Industry and Information Technology of China (Grant No. MIIT [2019]358) and the Ministry of Education of the People's Republic of China University-Industry Collaborative Education Program.

\section{References}

1. Deng R 2020 Reflection on the science and technology ethics of artificial intelligence development J. Guangxi Social Sciences 10 93-7

2. Yu X and Duan W 2019 Ethical construction of artificial intelligence $J$. Theoretical Exploration 06 43-9

3. He J and Meng P 2020 Xi Jinping's "five theories" on science and technology ethics J. Truth from Facts $\mathbf{3}$ 11-6

4. Zheng Z 2021 Ethical crisis and legal regulation of artificial intelligence algorithms J. Law Science. Journal of Northwest University of Political Science and Law 1 1-13

5. Turner J M 2019 Robot Rules: Regulating Artificial Intelligence (Switzerland: Palgrave Macmillan imprint)

6. Liu X 2020 Legal philosophical thoughts on the nature of tools of intelligent robots J. Criminal Science 5 20-34
7. Tang L 2020 Public nuisance to algorithm application and its governance paths $J$. Northern Legal Science 3 51-60.

8. Chen S 2020 Algorithmic governance: risks and countermeasures of technological alienation in intelligent society J. Journal of Hubei University. Philosophy and Social Sciences Edition 1 158-65

9. Luciano F M 2016 The 4th Revolution: How the infosphere is reshapin (Zhejiang: Zhejiang People's Publishing House) 